\title{
SUBMERSIONS FROM ANTI-DE SITTER SPACE WITH TOTALLY GEODESIC FIBERS
}

\author{
MARTIN A. MAGID
}

\section{Introduction}

In [5] O'Neill introduced the notion of a Riemannian submersion. Escobales [1], [2] classified Riemannian submersions from a sphere $S^{n}$ and from a complex projective space $\mathbf{C} P^{n}$ with totally geodesic fibers.

This paper investigates such submersions for an indefinite space form: anti-de Sitter space. It is shown that there is essentially only one submersion from $H_{1}^{2 n+1}$ onto a Riemannian manifold with totally geodesic fibers, and this is the standard one onto a complex hyperbolic space $\mathrm{CH}^{n}$.

1. Let $M, B$ be $C^{\infty}$ indefinite Riemannian manifolds. An indefinite Riemannian submersion $\pi: M \rightarrow B$ is an onto, $C^{\infty}$ mapping such that

(1) $\pi$ is of maximal rank,

(2) $\pi_{*}$ preserves the lengths of horizontal vectors, i.e., vectors orthogonal to the fibers $\pi^{-1}(x), x \in B$,

(3) the restriction of the metric to the vertical vectors is nondegenerate.

Consider the following example, [4, p. 282, Example 10.7] $p: H_{1}^{2 n+1} \rightarrow$ $\mathrm{CH}^{n}$, where $H_{1}^{2 n+1}$ is a $(2 n+1)$-dimensional anti-de Sitter space with constant sectional curvature -1 and signature $(1,2 n)$, and $\mathbf{C H}{ }^{n}$, defined below, is a complex hyperbolic space. On $\mathbf{C}^{n+1}$ let

$$
\begin{aligned}
& (\vec{z}, \vec{w})=-z_{0} \bar{w}_{0}+\sum_{k=1}^{n} z_{k} \bar{w}_{k}, \\
& \langle\vec{z}, \vec{w}\rangle=\operatorname{Re}(\vec{z}, \vec{w})=-x_{0} u_{0}-y_{0} v_{0}+\sum_{k=1}^{n} x_{k} u_{k}+y_{k} v_{k},
\end{aligned}
$$

where

$$
\begin{aligned}
\vec{z} & =\left(z_{0}, \cdots, z_{n}\right)=\left(x_{0}+i y_{0}, \cdots, x_{n}+i y_{n}\right), \\
\vec{w} & =\left(w_{0}, \cdots, w_{n}\right)=\left(u_{0}+i v_{0}, \cdots, u_{n}+i v_{n}\right), \\
H_{1}^{2 n+1} & =\left\{\vec{z} \in \mathbf{C}^{n+1}:(\vec{z}, \vec{z})=-1=\langle\vec{z}, \vec{z}\rangle\right\} \\
& =\left\{\left(x_{0}, y_{0}, \cdots, x_{n}, y_{n}\right):-x_{0}^{2}-y_{0}^{2}+x_{1}^{2}+\cdots+x_{n}^{2}+y_{n}^{2}=-1\right\} .
\end{aligned}
$$

Received May 23, 1980, and, in revised form, June 20, 1981. 
The tangent space to $H_{1}^{2 n+1}$ at $\vec{z}, T_{\vec{z}}$ is

$$
T_{\vec{z}}=\left\{W \in \mathbf{C}^{n+1}:\langle\vec{z}, W\rangle=0\right\} .
$$

Let $T_{\vec{z}}^{\prime}=\left\{U \in \mathbf{C}^{n+1}:\langle U, \vec{z}\rangle=0=\langle U, i \vec{z}\rangle\right\}$, and setting $H_{1}^{1}=\{\lambda \in \mathbf{C}$ : $\lambda \bar{\lambda}=1\}$ we have an $H_{1}^{1}$ action on $H_{1}^{2 n+1}, \vec{z} \mapsto \lambda \vec{z}$.

At each point of $H_{1}^{2 n+1}$ the vector field $i \vec{z}$ is tangent to the flow of the action, and $\langle i \vec{z}, i \vec{z}\rangle=-1$. Note that the orbit is $x_{t}=(\cos t+i \sin t) \vec{z}$ and $d x_{t} / d t=i x_{t}$. The orbit lies in the negative definite plane spanned by $\{\vec{z}, i \vec{z}\}$. The identification space of this action is called $\mathrm{CH}^{n}$, and the projection is denoted by $p$. It is easy to see that $T_{p(z)}\left(\mathrm{CH}^{n}\right)$ can be identified with $T_{z}^{\prime}$. This construction mimics that of $\mathbf{C} P^{n}$. $\mathbf{C} H^{n}$ has negative constant holomorphic sectional curvature. $p: H_{1}^{2 n+1} \rightarrow \mathbf{C} H^{n}$ is an indefinite Riemannian submersion.

The main result of this paper is

Theorem 1. If $\pi: H_{1}^{k} \rightarrow B^{j}$ is an indefinite Riemannian submersion from anti-de Sitter space to a Riemannian manifold with totally geodesic fibers, then $k=2 n+1, j=2 n$, and $B^{2 n}$ is holomorphically isometric to $\mathbf{C H}$, where $B^{j}$ is equipped with an integrable almost complex structure induced from the submersion. (See [1], [2].)

2. This section deals with the algebraic preliminaries.

Given $\pi: M \rightarrow B$, an indefinite Riemannian submersion, let $V$ and $H$ denote the vertical and horizontal projections.

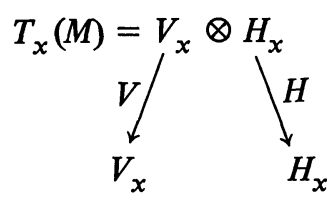

O'Neill [5] defines two fundamental tensors on $(M, \nabla,\langle\rangle$,$) :$

$A_{E} F=V\left(\nabla_{H E} H F\right)+H\left(\nabla_{H E} V F\right), \quad T_{E} F=H\left(\nabla_{V E} V F\right)+V\left(\nabla_{V E} H F\right)$,

for vector fields $E, F$ on $M$. These two tensors have the following properties:

(i) $A_{H E}=A_{E} ; T_{V E}=T_{E}$.

(ii) $A_{E}$ and $T_{E}$ are skew-symmetric with respect to $\langle$, $\rangle$.

(iii) $A_{E}$ and $T_{E}$ take vertical vectors to horizontal vectors and vice-versa.

(iv) If $V$ and $W$ are vertical and $X$ and $Y$ are horizontal, then

$$
T_{V} W=T_{W} V, \quad A_{Y} X=-A_{X} Y .
$$

Definition. A vector field $X$ on $M$ is said to be basic if it is the unique horizontal lift of a vector field $X_{*}$ on $B$, so that $\pi_{*}(X)=X_{*}$. 
Lemma $1[5, p .460]$. If $X$ and $Y$ are basic vector fields on $M$, then

(1) $\langle X, Y\rangle=\left\langle X_{*}, Y_{*}\right\rangle \cdot \pi$,

(2) $H[X, Y]$ is the basic vector field corresponding to $\left[X_{*}, Y_{*}\right]$,

(3) $H\left(\nabla_{X} Y\right)$ is the basic vector field corresponding to $\nabla_{X_{*}^{*}}^{*} Y_{*}$ where $\nabla^{*}$ is the connection on $B$.

Lemma $2[5, p .461]$. If $\nabla$ is the connection on $M$, and $\hat{\nabla}$ the connection on a fiber, then for $X, Y$ horizontal vector fields and $V, W$ vertical vector fields we have

(1) $\nabla_{V} W=T_{V} W+\hat{\nabla}_{V} W$,

(2) $\nabla_{V} X=H\left(\nabla_{V} X\right)+T_{V} X$,

(3) $\nabla_{X} V=A_{X} V+V\left(\nabla_{X} V\right)$,

(4) $\nabla_{X} Y=H\left(\nabla_{X} Y\right)+A_{X} Y$,

(5) if $X$ is basic, then $H\left(\nabla_{V} X\right)=A_{X} V$.

We will assume that the fibers are totally geodesic, so that by (1) $T_{V} W=0$, which gives

$(1)^{\prime} \nabla_{V} W=\hat{\nabla}_{V} W$

(2) $\nabla_{X} V=H\left(\nabla_{V} X\right)$.

O'Neill also proves $[5$, p. 465] the following relations between the sectional curvatures $K$ of $M$ and $K_{*}$ of $B$ when the fibers are totally geodesic:

$$
\begin{gathered}
K_{X \wedge V}=\frac{\left\langle A_{X} V, A_{X} V\right\rangle}{\langle X, X\rangle\langle V, V\rangle}, \\
K_{* X_{*} \wedge Y_{*}=} K_{X \wedge Y}+\frac{3\left\langle A_{X} Y, A_{X} Y\right\rangle}{\langle X, X\rangle\langle Y, Y\rangle-\langle X, Y\rangle^{2}},
\end{gathered}
$$

where $X$ and $Y$ are horizontal vector fields, $V$ is a vertical vector field, and $K_{E \wedge F}$ (respectively, $K_{* E_{*} \wedge F_{*}}$ ) denotes the sectional curvature in $M$ (respectively $B)$ of the plane spanned by $E$ and $F\left(E_{*}\right.$ and $\left.F_{*}\right)$.

In the Riemannian case, $(\theta \theta)$ says that sectional curvatures are increased by submersions. Since we will be dealing with submersions from $H_{1}^{m+k}$, let us first look at the case of submersion from a Lorentzian manifold with negative sectional curvature to a Riemannian manifold.

Proposition 1. If $\pi: M_{1}^{m+k} \rightarrow B^{m}$ is an indefinite Riemannian submersion with totally geodesic fibers, where $M$ is Lorentzian and has negative sectional curvature and $B$ is Riemannian, then $k=1$.

Proof. By $(\theta)$ we have

$$
0>K_{X \wedge V}=\frac{\left\langle A_{X} V, A_{X} V\right\rangle}{\langle X, X\rangle\langle V, V\rangle}
$$

Since $A_{X} V$ and $X$ are horizontal, $\left\langle A_{X} V, A_{X} V\right\rangle \geqslant 0$ and $\langle X, X\rangle>0$. Thus $\langle V, V\rangle\left\langle 0\right.$, i.e., $V$ is timelike, and $A_{X} V \neq 0$ for all horizontal $X \neq 0$, and all 
vertical $V \neq 0$. Since $M$ is Lorentzian, the timelike vectors are essentially one-dimensional and so the vertical vectors are one-dimensional. q.e.d.

Thus if $\pi: H_{1}^{m+1} \rightarrow B^{m}$ is a submersion with totally geodesic fibers, then by $(\theta \theta)$ we have

$$
K_{* X_{*} \wedge Y_{*}}=-1+\frac{3\left\langle A_{X} Y, A_{X} Y\right\rangle}{\langle X, X\rangle\langle Y, Y\rangle-\langle X, Y\rangle^{2}},
$$

and because $A_{X} Y$ is vertical, $\left\langle A_{X} Y, A_{X} Y\right\rangle \leqslant 0$. This shows that $K_{*} \leqslant-1$ so that curvature is nonincreasing in a submersion of this type.

Proposition 2. If $\pi: H_{1}^{m+1} \rightarrow B^{m}$ is a submersion with totally geodesic fibers, then $\pi_{j}\left(B^{m}\right)=0, j=1,2,3, \cdots$.

Hint of proof. We must only show that in the fibration

$$
S^{1} \stackrel{i}{\rightarrow} S^{1} \underset{H_{1}^{m+1}}{\times \mathbf{R}^{m}} \rightarrow B^{m}
$$

that $i$ induces a homotopy equivalence. This is clear, since every geodesic in $H_{1}^{m+1}$ is a circle in $\mathbf{R}_{2}^{m+2}$ of the form $(\cos t) x_{0}+(\sin t) X_{0}$ with $\left\langle x_{0}, X_{0}\right\rangle=0$.

Theorem 2. If $\pi: H_{1}^{m+1} \rightarrow B^{m}$ is an indefinite Riemannian submersion with totally geodesic fibers, then $m=2 n$, for some $n>0$.

Proof. $H_{1}^{m+1}$ is not only equipped with the fundamental tensor $A$ but also with a foliation by timelike geodesics. Thus there is a smooth vector field $V$ tangent to these geodesics with $\langle V, V\rangle=-1$. Let $X$ and $Y$ be horizontal vector fields on $H_{1}^{m+1}$. We know that $A_{X} V$ is horizontal. Therefore

$$
0=Y\langle X, V\rangle=\left\langle\nabla_{Y} X, V\right\rangle+\left\langle X, \nabla_{Y} V\right\rangle=\left\langle A_{Y} X, V\right\rangle+\left\langle X, A_{Y} V\right\rangle \text {. }
$$

Interchanging $X$ and $Y$ we have

$$
0=\left\langle A_{X} Y, V\right\rangle+\left\langle Y, A_{X} V\right\rangle .
$$

Since $A_{X} Y+A_{Y} X=0$, adding these two equations yields

$$
\left\langle X, A_{Y} V\right\rangle+\left\langle Y, A_{X} V\right\rangle=0,
$$

so that $A_{-} V: H_{x} \rightarrow H_{x}$ is skew-symmetric. If the horizontal space $H_{x}$ were odd dimensional, then $A_{-} V$ would have 0 as an eigenvalue. On the other hand, $(\theta)$ gives

$$
\frac{\left\langle A_{X} V, A_{X} V\right\rangle}{\langle X, X\rangle\langle V, V\rangle}=-1
$$

But $\langle V, V\rangle=-1$, so $\left\langle A_{X} V, A_{X} V\right\rangle=\langle X, X\rangle$ which means $A_{-} V$ is an isometry. Thus $H_{x}$ must be even dimensional, and $m=2 n$. q.e.d. 
In fact a skew-symmetric isometry is an almost complex structure, since a basis can be found with respect to which the mapping is of the form

$$
\left[\begin{array}{rrrr}
0 & 1 & & \\
-1 & 0 & & \\
& & 0 & 1 \\
& & -1 & 0
\end{array}\right]
$$

Thus we know that any indefinite Riemannian submersion from $H_{1}^{k}$ with totally geodesic fibers onto a Riemannian manifold is of the form $\pi: H_{1}^{2 n+1}$ $\rightarrow B^{2 n}$, and $B^{2 n}$ is simply connected.

3. This part of the paper will show that $B^{2 n}$ is holomorphically isometric to $D^{n}$, the disc in $\mathbf{C}^{n}$ with the Bergman metric [4, Ex. 10.7].

First we shall show that the submersion induces an almost complex structure on $B^{2 n}$ and a Hermitian metric on $B^{2 n}$. Then it will be seen that with these induced structures $B^{2 n}$ is a Kähler manifold.

One could also show that $H_{1}^{2 n+1}$ is an indefinite regular Sasakian manifold with the structure induced from the submersion and so [6, p. 150] $B^{2 n}$ is a real $2 n$-dimensional Kähler manifold. The proofs are similar.

Let $V$ be as in the proof of Theorem 2. Since $V$ is a geodesic vector field, $\nabla_{V} V=0$. Let $\phi(E)=A_{E} V$ for all vector fields $E$ on $H_{1}^{2 n+1}$, and let $\eta$ be the one-form dual to $V$, so that $\eta(V)=-1$. Then we have

Lemma 3. (1) $\phi(V)=0$,

(2) $\eta(\phi(E))=0$,

(3) $\phi^{2}(E)=-E-\eta(E) V$,

(4) $\langle\phi(E), \phi(F)\rangle=\langle E, F\rangle+\eta(E) \eta(F)$,

(5) $\eta(E)=\langle E, V\rangle$,

for all vector fields $E, F$ on $H_{1}^{2 n+1}$.

Proof. (1), (2), (5) are clear.

(3) Let $E=X+\lambda V$ where $X$ is horizontal. Then

$$
\phi^{2}(E)=A_{A_{E} V} V=A_{A_{X} V} V \text {, and } A_{A_{X} V} V=-X,
$$

since for all horizontal $Y$

$$
\begin{aligned}
\left\langle A_{A_{X} V} V, Y\right\rangle & =-\left\langle V, A_{A_{X} V} Y\right\rangle=\left\langle V, A_{Y} A_{X} V\right\rangle \\
& =-\left\langle A_{Y} V, A_{X} V\right\rangle=-\langle X, Y\rangle .
\end{aligned}
$$

Thus

$$
\phi^{2}(X+\lambda V)=-X=-(X+\lambda V)-\eta(X+\lambda V) V=-E-\eta(E) V .
$$

(4) Let $E=X+\lambda V, F=Y+\mu V$ where $X$ and $Y$ are horizontal. Then

$$
\begin{aligned}
\langle\phi E, \phi F\rangle & =\left\langle A_{E} V, A_{F} V\right\rangle=\left\langle A_{X} V, A_{Y} V\right\rangle \\
& =\langle X, Y\rangle=\langle X+\lambda V, Y+\mu V\rangle+\eta(X+\lambda V) \eta(Y+\mu V) .
\end{aligned}
$$


Since the basic vector fields on $H_{1}^{2 n+1}$ correspond to vector fields on $B^{2 n}$, we focus our attention on these vector fields. In particular, in order to have $\phi$ induce an almost complex structure on $B^{2 n}$, if $X$ is basic, then $A_{X} V$ must be basic.

Theorem 3. If $X$ is a basic vector field on $H_{1}^{2 n+1}$, then $A_{X} V$ is a basic vector field.

Proof. Lemma 1.2 [1, p. 254]: Let $B_{i}$ be a basic vector field on $H_{1}^{2 n+1}$ corresponding to $B_{i_{*}}$ on $B^{2 n}$, and let $X$ be horizontal. If $\left\langle X, B_{i}\right\rangle_{p}=\left\langle X, B_{i}\right\rangle_{p^{\prime}}$ for all such $B_{i}$ and any $p, p^{\prime}$ in $\pi^{-1}(b), b \in B^{2 n}$, then $X$ is basic.

This means that for all $B$, basic, we must show that $V\left\langle A_{X} V, B\right\rangle=0$. Since

$$
\begin{aligned}
V\left\langle A_{X} V, B\right\rangle & =\left\langle\nabla_{V}\left(A_{X} V\right), B\right\rangle+\left\langle A_{X} V, \nabla_{V} B\right\rangle \\
& =\left\langle\nabla_{V}\left(A_{X} V\right), B\right\rangle+\left\langle A_{X} V, A_{B} V\right\rangle \\
& =\left\langle\nabla_{V}\left(A_{X} V\right), B\right\rangle+\langle X, B\rangle,
\end{aligned}
$$

we must show that for $X$ basic $\nabla_{V}\left(A_{X} V\right)=-X$. On $H_{1}^{2 n+1}$

$$
R(V, X) V=\nabla_{V} \nabla_{X} V-\nabla_{X} \nabla_{V} V-\nabla_{[X, V]} V=-(V \wedge X) V,
$$

since $H_{1}^{2 n+1}$ has constant curvature -1 .

$R(V, X) V=\nabla_{V} \nabla_{X} V-\nabla_{[X, V]} V$ since $\nabla_{V} V=0$, and because $[V, X]$ is vertical $\nabla_{[X, V]} V=\rho \nabla_{V} V=0$ yielding $R(V, X) V=\nabla_{V} \nabla_{X} V$.

On the other hand

$$
R(V, X) V=-(\langle X, V\rangle V-\langle V, V\rangle X)=-X
$$

so $\nabla_{V} \nabla_{X} V=-X$. But

$$
\nabla_{V}\left(\nabla_{X} V\right)=\nabla_{V}\left(A_{X} V+V\left(\nabla_{X} V\right)\right)=\nabla_{V}\left(A_{X} V\right)
$$

since $\left\langle\nabla_{X} V, V\right\rangle=\frac{1}{2} X\langle V, V\rangle=0$. q.e.d.

Thus $\phi$ induces an almost complex structure on $B^{2 n}$.

Theorem 4. This almost complex structure on $B^{2 n}$ is integrable.

Proof. We must show that $N_{\phi}\left(X_{*}, Y_{*}\right)=0$ where $X_{*}$ and $Y_{*}$ are vector fields on $B^{2 n}$, and $N_{\phi}$ is the Nijenhuis tensor of $\phi$ :

$$
N_{\phi}\left(X_{*}, Y_{*}\right)=\left[\phi X_{*}, \phi Y_{*}\right]-\left[X_{*}, Y_{*}\right]-\phi\left[X_{*}, \phi Y_{*}\right]-\phi\left[\phi X_{*}, Y_{*}\right]
$$

The basic vector field corresponding to $N_{\phi}\left(X_{*}, Y_{*}\right)$ is $H[\phi X, \phi Y]-H[X, Y]$ - $\phi[X, \phi Y]-\phi[\phi X, Y]$ where $X$ and $Y$ are the basic vector fields associated 
with $X_{*}$ and $Y_{*}$. This is equivalent to

$$
\begin{aligned}
& H\left(\nabla_{\phi X} \phi Y\right)-H\left(\nabla_{\phi Y} \phi X\right)-H\left(\nabla_{X} Y\right)+H\left(\nabla_{Y} X\right)-\phi\left(\nabla_{X} \phi Y\right) \\
& +\phi\left(\nabla_{\phi Y} X\right)-\phi\left(\nabla_{\phi X} Y\right)+\phi\left(\nabla_{Y} \phi X\right) \\
& \text { (a) (b) (c) (d) } \\
& =H\left(\nabla_{\left(A_{X} V\right)}\left(A_{Y} V\right)\right)-H\left(\nabla_{\left(A_{Y} V\right)}\left(A_{X} V\right)\right)-H\left(\nabla_{X} Y\right)+H\left(\nabla_{Y} X\right) \\
& =A_{\nabla_{X}\left(A_{Y} V\right)}^{(e)} V+A_{\nabla_{\left(A_{Y} V\right)^{X}}}^{(f)} V-A_{\nabla_{\left(A_{X} V\right.} Y}^{(g)} V+A_{\nabla_{Y}\left(A_{X} V\right)}^{(h)} V .
\end{aligned}
$$

In order to prove $N_{\phi}\left(X_{*}, Y_{*}\right)=0$ it is sufficient to prove

Lemma 4. If $X$ and $Y$ are horizontal vector fields on $H_{1}^{2 n+1}$, then

$$
H\left(\nabla_{X}\left(A_{Y} V\right)\right)=A_{\left(\nabla_{X} Y\right)} V
$$

If $(\dagger)$ holds, then

$$
\begin{gathered}
H\left(\nabla_{A_{X} V} A_{Y} V\right)=A_{\nabla_{A_{X} V} V} V, \\
H\left(\nabla_{A_{Y} V} A_{X} V\right)=A_{\nabla_{A_{Y} V} X} V \\
A_{\nabla_{X}\left(A_{Y} V\right)} V=H\left(\nabla_{X}\left(A_{A_{Y} V} V\right)\right)=-H\left(\nabla_{X} Y\right), \\
A_{\nabla_{Y}\left(A_{X} V\right.} V=-H\left(\nabla_{Y} X\right),
\end{gathered}
$$

and so $(a)=(g),(b)=(f),(e)=-(c)$ and $(h)=-(d)$. Thus the sum is zero.

Proof of Lemma 4. ( $\dagger$ ) is equivalent to

$$
\left\langle\nabla_{X} A_{Y} V, Z\right\rangle=\left\langle A_{\nabla_{\underline{X}} Y} V, Z\right\rangle \text { for all horizontal } Z \text {. }
$$

From $[5$, p. $464\{3\}]$

$$
\langle R(Y, Z) X, V\rangle=-\left\langle\left(\nabla_{X} A\right)_{Y} Z, V\right\rangle,
$$

so

$$
\langle R(Y, Z) V, X\rangle=\left\langle\left(\nabla_{X} A\right)_{Y} Z, V\right\rangle
$$

Since $R(Y, Z) V=-(Y \wedge Z) V=0$, we have $\left\langle\left(\nabla_{X} A\right)_{Y} Z, V\right\rangle=0$, which expands to

$$
0=\left\langle\nabla_{X}\left(A_{Y} Z\right), V\right\rangle-\left\langle A_{\nabla_{X} Y} Z, V\right\rangle-\left\langle A_{Y}\left(\nabla_{X} Z\right), V\right\rangle .
$$

Substituting

$$
A_{Y} Z=-\left\langle A_{Y} Z, V\right\rangle V=\left\langle A_{Y} V, Z\right\rangle V
$$


in the above equation gives

$$
\begin{aligned}
0= & \left\langle\nabla_{X}\left\langle A_{Y} V, Z\right\rangle V, V\right\rangle-\left\langle A_{\nabla_{X} Y} Z, V\right\rangle-\left\langle A_{Y}\left(\nabla_{X} Z\right), V\right\rangle \\
= & \left\langle A_{Y} V, Z\right\rangle\left\langle\nabla_{X} V, V\right\rangle+\left\langle X\left\langle A_{Y} V, Z\right\rangle V, V\right\rangle \\
& -\left\langle A_{\nabla_{X} Y} Z, V\right\rangle-\left\langle A_{Y}\left(\nabla_{X} Z\right), V\right\rangle \\
= & -\left\langle\nabla_{X}\left(A_{Y} V\right), Z\right\rangle-\left\langle A_{Y} V, \nabla_{X} Z\right\rangle-\left\langle A_{\nabla_{X} Y} Z, V\right\rangle-\left\langle A_{Y}\left(\nabla_{X} Z\right), V\right\rangle \\
= & \left\langle\nabla_{X}\left(A_{Y} V\right), Z\right\rangle+\left\langle A_{Y} V, \nabla_{X} Z\right\rangle-\left\langle Z, A_{\nabla_{X} Y} V\right\rangle+\left\langle A_{Y}\left(\nabla_{X} Z\right), V\right\rangle \\
= & \left\langle\nabla_{X}\left(A_{Y} V\right), Z\right\rangle-\left\langle A_{\nabla_{X} Y} V, Z\right\rangle
\end{aligned}
$$

because $\left\langle A_{Y} V, \nabla_{X} Z\right\rangle+\left\langle A_{Y}\left(\nabla_{X} Z\right), V\right\rangle=0$. q.e.d.

Note that the metric induced on $B^{2 n}$ is Hermitian since $\langle\phi X, \phi Y\rangle=$ $\langle X, Y\rangle$ for $X, Y$ basic on $H_{1}^{2 n+1}$. Thus in order to show that $B^{2 n}$ is Kählerian we must only show that

$$
\nabla_{X_{*}}^{*} \phi Y_{*}=\phi\left(\nabla_{X_{*}}^{*} Y_{*}\right)
$$

Since the basic vector field corresponding to $\nabla_{X_{*}}^{*} Y_{*}$ is $H\left(\nabla_{x} Y\right)$ and the basic vector field corresponding to $\nabla_{X_{*}^{*}}^{*} Y_{*}$ is $H\left(\nabla_{X} \phi Y\right)$, we must show that

$$
H\left(\nabla_{X} \phi Y\right)=\phi\left(\nabla_{X} Y\right)
$$

for $X, Y$ basic on $H_{1}^{2 n+1}$. But this is just $(\dagger)$.

Thus $B^{2 n}$ is a Kähler manifold, $\pi_{1}\left(B^{2 n}\right)=0$ and to finish the proof of Theorem 1 it is only necessary to show that $B^{2 n}$ has constant holomorphic sectional curvature [4, p. 170, Theorem 7.9].

By $(\theta \theta)$ we obtain

$$
\begin{aligned}
K_{* X_{*} \wedge \phi X_{*}}= & K_{X \wedge \phi X}+3 \frac{\left\langle A_{X} \phi X, A_{X} \phi X\right\rangle}{\langle X, X\rangle\langle\phi X, \phi X\rangle-\langle X, \phi X\rangle^{2}} \\
= & -1+3 \frac{\left\langle A_{X} A_{X} V, A_{X} A_{X} V\right\rangle}{\langle X, X\rangle^{2}}
\end{aligned}
$$

Note $A_{X} A_{X} V=-\left\langle A_{X} A_{X} V, V\right\rangle V=\left\langle A_{X} V, A_{X} V\right\rangle V=\langle X, X\rangle V$, so that

$$
K_{* X_{*} \wedge \phi X_{*}}=-1+3 \frac{\langle X, X\rangle^{2}\langle V, V\rangle}{\langle X, X\rangle^{2}}=-4 .
$$

This completes the proof of Theorem 1 .

Just as Escobales does in [1] we can show that any two such maps are equivalent. 


\section{Bibliography}

[1] R. H. Escobales, Jr., Riemannian submersions with totally geodesic fibers, J. Differential Geometry 10 (1975) 253-276.

[2] _ Riemannian submersions from complex projective space, J. Differential Geometry 13 (1978) 93-107.

[3] A. Gray, Pseudo Riemannian almost product manifolds and submersions, J. Math. Mech. 16 (1967) 715-737.

[4] S. Kobayashi \& K. Nomizu, Foundations of Differential Geometry, Vol. II, Interscience, New York, 1969.

[5] B. O'Neill, The fundamental equations of a submersion, Mich. Math. J. 13 (1966) 459-469.

[6] K. Yano \& M. Kon, Anti-invariant submanifolds, Marcel Dekker, New York, 1976.

Wellesley College

UNIVERSITY OF CALIFORNIA, BERKELEY 
